\title{
Calidad de vida y apoyo social en mujeres con cáncer de mama
}

\author{
Quality of life and social support in women with breast cancer
}

\author{
Qualidade de vida e apoio social em mulheres com câncer de mama
}

\section{Recibido: \\ 5 Diciembre de 2016 \\ Aprobado: \\ 31 Mayo de 2017}

Autor de correspondencia

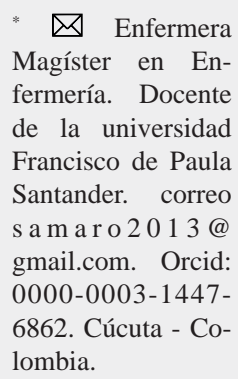

\author{
Sandra Milena Martínez Rojas*
}

\section{Resumen}

Objetivo: Establecer la relación entre calidad de vida y apoyo social en mujeres diagnosticadas con cáncer de mama que reciben tratamiento de quimioterapia en una clínica cancerológica de Norte de Santander. Materiales y Métodos: Estudio cuantitativo correlacional, de tipo descriptivo transversal, en el cual se calculó una muestra de 120 mujeres, con una selección a conveniencia intencionada. La recolección de la información se realizó por medio de los instrumentos de QOL de Betty Ferrell para evaluar la calidad de vida y SSCI de G. Hilbert para el apoyo social de este grupo de mujeres. Resultados: Se presentó una calidad de vida buena, en donde las dimensiones que más se correlacionaron fueron la física y la psicológica. Así mismo, el mayor apoyo social se obtuvo de las personas que las rodean, evidenciándose la relación significativa de todas las dimensiones entre sí. Conclusión: Al establecer la relación entre calidad de vida y apoyo social en las mujeres con cáncer de mama que reciben tratamiento con quimioterapia, se acepta la hipótesis nula de que no existe correlación entre las dos variables, aunque el valor $p,=0,002$ representa una significancia estadística. Se deduce que las dos variables, aunque son independientes, se relacionan entre sí por las dimensiones y las sub-escalas.

Palabras claves: Calidad de vida, apoyo social y enfermedad crónica

\section{Abstract}

Objective: To establish the relationship between quality and social support in women diagnosed with breast cancer that chemotherapy treatment in the cancer clinic of Norte de Santander. Materials and Methods: Correlational quantitative study, of transversal descriptive type, in which a sample of 120 women was calculated, with a selection of intentional convenience. The collection of the information was done through the instruments of QOL of Betty Ferrell and (SSCI) of G. Hilbert where the quality of life and social support of this group of women was established. Results: A quality-life relationship was presented in the dimensions that most correlated with the physical and the psychological, likewise, social support was obtained from the people around them, evidencing the significant relationship of all the dimensions among themselves. Conclusion: the relationship between quality of life and social support in women with breast cancer and treatment with chemotherapy, accepts the hypothesis that there is no correlation between the variables, although the $\mathrm{p}$ value, $=0.002$ represents a statistical significance, It follows that the two variables, although independent, are related to each other by the dimensions and subscales.

Key words: Quality of life, social support and chronic disease

\section{Resumo}

Objetivo: Estabelecer a relação entre qualidade de vida e apoio social em mulheres diagnosticadas com câncer de mama que recebem tratamento de quimioterapia numa clínica de câncer no estado Norte de Santander-Colômbia. Materiais e Métodos: Estudo quantitativo correlacional de tipo

\section{Para citar este artículo/ To reference this article/ Para citar este artigo/}

Martínez Rojas SM. Significados de ser portadoras de hemofilia. Rev. cienc. cuidad. 2018; 15(1):34-47.

Este es un artículo bajo la licencia CC BY (https://creativecommons.org/licenses/by/4.0/) @ ) 
descritivo transversal, no qual se calculou uma amostra de 120 mulheres, com uma seleção a conveniência intencionada. A recolecção da informação foi feita por meio dos instrumentos de QOL de Betty Ferrell para avaliar a qualidade de vida e SSCI de G. Hilbert para o apoio social deste grupo de mulheres. Resultados: Apresentou-se uma boa qualidade de vida, onde as dimensões que mais se correlacionaram foram a física e a psicológica. Do mesmo modo, o maior apoio social foi obtido junto das pessoas que os rodeiam, evidenciando-se a relação significativa de todas as dimensões entre si. Conclusão: Ao estabelecer a relação entre qualidade de vida e apoio social nas mulheres com câncer de mama que recebem tratamento com quimioterapia, a hipótese nula aceita que não existe correlação entre as duas variáveis, embora o valor $\mathrm{p},=0,002$ represente uma significância estatística. Pode deduzir-se que as duas variáveis, embora sejam independentes, se relacionam entre si pelas dimensões e as sub-escalas.

Palavras-chave: Apoio social, doença crônica, qualidade de vida

\section{Introducción}

Según la Organización Mundial de la Salud (OMS), el cáncer figura entre las principales causas de muerte en todo el mundo, debido al resultado de la interacción entre los factores genéticos de una persona y tres categorías de agentes externos, que incluyen: carcinógenos físicos tales como la radiación ultravioleta e ionizante, carcinógenos químicos como el asbesto y el humo del tabaco, y carcinógenos biológicos como las infecciones de ciertos virus, bacterias o parásitos (1), destacándose la mujer como el género más afectado por esta enfermedad, en especial por el cáncer de mama.

El cáncer implica una situación de vida que genera cambios físicos, psicológicos y sociales; así mismo, a nivel personal, familiar y social, afecta la calidad de vida en este grupo de mujeres, para quienes resulta fundamental el apoyo social para cubrir las necesidades generadas por esta enfermedad.

Dada la importancia de esta problemática, se reconoce que, al contar con una red de apoyo conformada por familiares, vecinos, amigos y sistema de salud, las mujeres podrán tener un refugio en las redes, consiguiendo de esta manera aliviar los cambios inesperados que se presentan por esta patología y mejorando su calidad de vida.

De esta forma, el apoyo social y la calidad de vida son fenómenos de gran importancia para el cuidado de la salud en la población que padece el cáncer de mama, puesto que genera seguridad, bienestar, tranquilidad y satisfacción en el proceso que está viviendo este grupo.
En el ámbito internacional, latinoamericano, nacional, regional y local se establece que el cáncer de mama es un problema de salud pública frecuente, de gran complejidad, seguido por el cáncer de pulmón y que constituye una de las primeras causas de muerte en las mujeres (2-3).

En Cúcuta, la mortalidad por tumores malignos en mama y próstata es concordante con los datos nacionales. En una clínica cancerológica de la capital nortesantandereana, las estadísticas patológicas fueron: tumor maligno de mama en parte no especificada, tumor maligno del cuadrante superior externo de la mama, carcinoma in situ de la mama y tumor maligno del pezón y/o areola mamaria, con un promedio mensual de 7 mujeres padeciendo esta enfermedad (4).

Los referentes teóricos para el desarrollo del estudio fueron: Ferrell (6-8) con su enfoque en los pacientes con enfermedades crónicas, teniendo en cuenta el abordaje del constructo calidad de vida de manera integral en los aspectos físicos, psicológicos, sociales y espirituales que están presentes en todas las enfermedades crónicas, y los postulados sobre el apoyo social de Hilbert (9), quien propone al respecto dimensiones como interacciones personales, guía, retroalimentación, ayuda tangible e interacción social.

Para Ferrell (5), la calidad de vida se define como una construcción multidimensional caracterizada por el bienestar en aspectos de la vida que son importantes para el individuo. Abarca la interacción de la salud y el funcionamiento psicológico, espiritual, 
ISSN-PRINT

1794-9831

E-ISSN 2322-7028

Vol. 15 No. 1

Ene - Jun 2018

Cúcuta, Colombia socioeconómico y familiar, a partir de cuatro dominios:

Bienestar físico: se define como la salud física en general y los síntomas que se presentan, entre los cuales se enuncian la fatiga, las alteraciones del sueño, la funcionalidad, las náuseas, las alteraciones en el apetito, el estreñimiento, los dolores y el malestar.

Bienestar psicológico: se toma como la búsqueda de un sentido de control en el enfrentamiento de una enfermedad que amenaza la vida, caracterizada por un problema emocional, alteración de prioridades de la vida y miedo a lo desconocido, al igual que cambios positivos en la vida.

Bienestar social: brinda la posibilidad de ver a la persona no sólo desde la enfermedad sino también a la persona que rodea a la misma, medio por el cual se reconoce al paciente con la enfermedad, sus relaciones y roles.

Bienestar espiritual: se enmarca dentro del significado de los acontecimientos, la habilidad para mantener la esperanza, la religiosidad o la trascendencia para obtener el sentido de la experiencia de la enfermedad, caracterizada por la incertidumbre.

De la misma manera, Hilbert considera el apoyo social como un concepto multidimensional del cual emergen otros conceptos como la red social, la interacción que existe entre los individuos que se encuentran inmersos en dicha red, los recursos que provee la fuente de soporte, la percepción de la persona frente al mismo y los aspectos emocionales e instrumentales. Este autor clasifica el soporte social en cinco categorías:

Interacción personal: representado en el apoyo de tipo emocional para el afrontamiento propicio de las diferentes situaciones de la enfermedad crónica.

La guía: todas aquellas ayudas que percibe la persona por medio de la educación y la información.

La retroalimentación: representada en los sentimientos acerca de quien brinda apoyo, el hecho de estar de acuerdo con esta persona y, además, con quienes apoyan los pensamientos y las acciones del enfermo.

La ayuda tangible: consistente en la ayuda material y en las tareas físicas y las labores que involucran el desempeño del enfermo en sus diferentes roles.
Interacción social: apoyo con el cual se pretende estimular la recuperación de las relaciones con otros, que contribuyan a dar respuesta a sus necesidades. (6) Para Enfermería, estos constructos comprenden las vivencias de la salud humana y generan validez para la construcción de nuevo conocimiento.

La presente investigación es una herramienta para el quehacer en enfermería, mejorando, a través del conocimiento, la práctica del enfermero sobre las redes de apoyo social y la calidad de vida frente a los problemas generados por la enfermedad.

La significancia teórica propone que los dos conceptos principales son reconocidos en el área de cuidado como fenómenos de interés que afectan a las personas y/o a los pacientes susceptibles del cuidado de enfermería.

La significancia social se orienta hacia el favorecimiento del desarrollo de redes de apoyo social y la calidad de vida en las mujeres con cáncer de mama.

Los estudios encontrados de estas dos variables reflejan la importancia de estudiar la particularidad de la mujer con cáncer de mama en tratamiento con quimioterapia en la población colombiana, como soporte teórico para la definición de las intervenciones de enfermería.

\section{Objetivos}

\section{Objetivo General}

Establecer la relación entre calidad de vida y apoyo social en mujeres diagnosticadas con cáncer de mama que reciben tratamiento de quimioterapia en una clínica cancerológica de Norte de Santander, en el año 2015

\section{Objetivo Específicos}

- Determinar la calidad de vida de las mujeres diagnosticadas con cáncer de mama que reciben tratamiento de quimioterapia en una clínica cancerológica de Norte de Santander, en el año 2015.

- Determinar el apoyo social de las mujeres diagnosticadas con cáncer de mama que reciben tratamiento de quimioterapia en una clínica cancerológica de Norte de Santander, en el año 2015. 
- Estimar la asociación entre calidad de vida y apoyo social en las mujeres diagnosticadas con cáncer de mama que reciben tratamiento de quimioterapia en una clínica cancerológica de Norte de Santander, en el año 2015.

\section{Materiales y Métodos}

La investigación se plantea desde un enfoque cuantitativo, de tipo descriptivo correlacional, de corte transversal, en el que participaron mujeres diagnosticadas con cáncer de mama que asistieron a la clínica cancerológica de Norte de Santander y que aceptaron participar de forma voluntaria, firmando el consentimiento informado.

Los criterios de inclusión fueron: mujeres mayores de 25 años diagnosticadas con cáncer de mama en cualquiera de sus estadios y que recibieran tratamiento con quimioterapia. Como criterios de exclusión se tuvieron: mujeres con alteraciones psiquiátricas, hospitalizadas en otros servicios, que presentaran otras patologías, estuvieran en el momento de la entrevista bajo efecto de medicamentos o sustancias que limitaran su habilidad para comunicarse.

Se contó con una muestra de 120 participantes, utilizando el método aleatorio simple, con un $95 \%$ de confiabilidad y un $5 \%$ en el error de estimación, utilizando la fórmula de muestreo probabilístico.

Se tomó en cuenta el perfil sociodemográfico, con datos como la edad, el estado civil, la convivencia de los participantes y otros tratamientos recibidos aparte de la quimioterapia, variables a tener en cuenta en el trascurso de la enfermedad.

Para la recolección de la información se utilizaron los instrumentos de calidad de vida versión familiar (QOL) de la autora Betty Ferrell, con una confiabilidad de 0.89 y una consistencia interna del alfa de cronbach de 0.69. El análisis factorial confirmó los 4 dominios de QOL como sub-escalas para el instrumento, el cual fue validado para población colombiana y consta de 41 ítems con respuesta en escala tipo de Likert, estructurado en cuatro dimensiones: Bienestar físico, Bienestar psicológico, Bienestar social y Bienestar espiritual. La escala de medición original del instrumento es de 0 a 10; sin embargo, en el presente estudio se aplicó la evaluación de cada ítem mediante una escala Likert de 1 a 4, donde 0 representa la peor y 4 la mejor percepción para cada ítem, adaptación realizada por el grupo de Docentes de la línea de investigación Cuidado al Paciente Crónico de la Facultad de Enfermería de la Universidad Nacional de Colombia (10).

Simultáneamente se aplicó el Inventario de apoyo social en enfermedad crónica (SSCI), de G. Hilbert, que contempla cinco sub-escalas: interacción personal, guía, retroalimentación, ayuda tangible e interacción social. En Colombia, el instrumento SSCI fue traducido por el grupo de cuidado al paciente crónico y su familia de la Facultad de Enfermería de la Universidad Nacional, con una aplicación inicial a 132 cuidadores de niños con enfermedad crónica, que reportó consistencia interna con un alfa de cronbach de 0,95 . Se evidenció que el SSCI es un instrumento confiable para ser aplicado en la población colombiana, en tanto consta de 38 preguntas: 10 ítems miden la dimensión Interacción personal, 14 la de guía, 5 la correspondiente a retroalimentaciones, 5 la de interacción social y 4 la de ayuda tangible. La puntuación se basó en una escala de 1 a 6 , en donde 1 corresponde a insatisfecho y 6 a muy satisfecho. Para la interpretación de los resultados, el inventario tuvo un puntaje mínimo de 38 y máximo de 228 , teniendo en cuenta que entre más alto es el puntaje, mayor es la satisfacción (11).

La información fue introducida a un programa Excel y posteriormente fue procesada por medio del programa SPSS versión 23 de 2014, con el propósito de obtener la información para el análisis de cada variable.

La presente investigación se desarrolló siguiendo los lineamientos éticos de la Resolución 008430 de octubre 4 de 1993, que en su artículo $2^{\circ}$ determina que "las Instituciones que vayan a realizar investigación en humanos, deberán tener un Comité de Ética en Investigación, encargado de resolver todos los asuntos relacionados en el tema", y en su artículo $6^{\circ}$ establece que "la investigación que se realice en seres humanos deberá desarrollarse conforme a los siguientes criterios, entre ellos: que se ajustará a los principios científicos y éticos que la justifiquen y que se llevará a cabo cuando se obtenga la aprobación del proyecto por parte del Comité de Ética en Investigación de la institución" (12). Por lo anterior, esta investigación se 
ISSN-PRINT

1794-9831

E-ISSN 2322-7028

Vol. 15 No. 1

Ene - Jun 2018

Cúcuta, Colombia presentó para su revisión y aval a la subcomisión de investigación de la Universidad de La Sabana.

\section{Resultados}

Se realizó la recolección de la información en el segundo semestre del año 2015. Se presentaron algunas limitaciones en el estudio al momento de la búsqueda de las mujeres, puesto que se requirió desplazarse muchas veces al domicilio de cada una cuando no asistían a la clínica cancerológica.

\section{Variables Sociodemográficas}

Respecto a la edad de las mujeres, se observa que, en su mayoría, están en la etapa de madurez. Llama la atención que un gran número de ellas se agrupó en el rango de edades de 40-59 años $(56 \%)$, mientras que apenas un $11 \%$ se encontró entre los 24 y 39 años, lo cual demuestra que el cáncer de mama también se presenta en mujeres jóvenes.
En relación con el estado civil, se resalta que la mayoría de las mujeres $(36.7 \%)$ eran casadas y solamente un $11 \%$ manifestó ser soltera.

En cuanto a la convivencia, se observó que, aunque la mayoría eran casadas, habitaban con otros familiares que no eran ni sus esposos ni sus hijos (48\%).

\section{Determinación de la calidad de vida en general y en sus cuatro dimensiones: bienestar físico, psicológico, social y espiritual:}

A continuación se presentan los hallazgos de la calidad de vida de las mujeres diagnosticadas con cáncer de mama a nivel general y con sus cuatro dimensiones, física, psicológica, social y espiritual.

En general, la calidad de vida de las mujeres con cáncer de mama presentó una ligera simetría, el puntaje mínimo fue de 77 puntos y el máximo fue de 140, la mediana fue de 113 puntos y se estableció una media de 111,683 con una desviación estándar de 12,34.

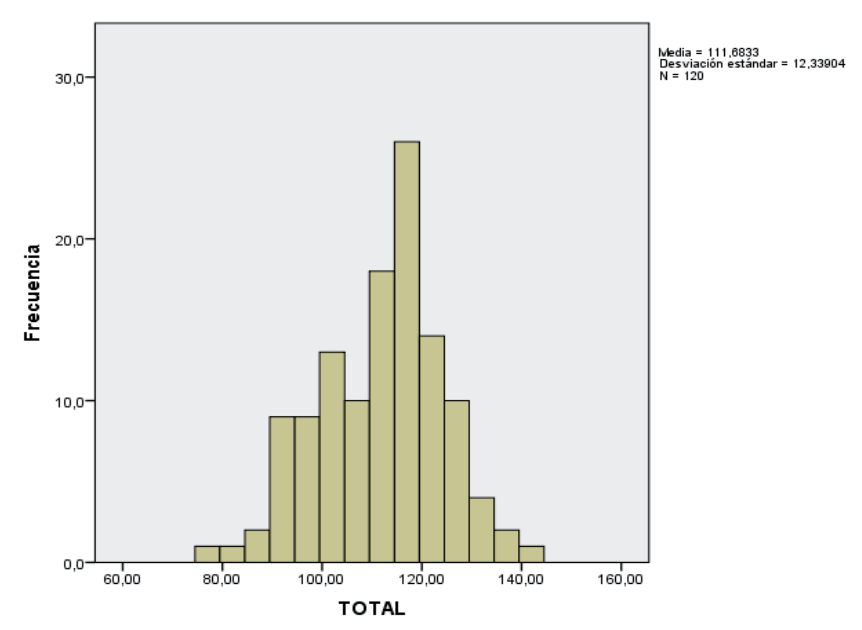

Gráfica 1. Histograma total calidad de vida pacientes con cáncer de mama

Fuente: Tesis Maestría Martínez, SM. (2015)

De este modo, las puntuaciones de la Escala Calidad de Vida, versión familiar, pueden fluctuar entre $41 \mathrm{y}$ 164. Como ha expresado Ferrell, la calidad de vida es multidimensional y comprende los dominios; bienestar físico, bienestar psicológico, bienestar social y bienestar espiritual. El rango de medición con el instrumento aplicado fue de 41 a 164, correspondiendo a 41 ítems, tal como se observa en la Gráfica 1. En el estudio, el Bienestar físico de las mujeres diagnosticadas con cáncer de mama reportó una media de 22.975, con una desviación estándar de 5.66 y con un valor máximo de 32 y mínimo de 9. 
En Bienestar Psicológico, la investigación reportó una media de 49,383, con desviación estándar de 8,55. La mediana fue 49 con rango de 40 y un valor máximo de 68 y mínimo de 28. Bienestar Social presentó una media de 19,083, con una desviación estándar de
2,9208 y con un valor máximo de 28 y mínimo de 13 .

El Bienestar Espiritual evidenció una media de 20,242, con una desviación estándar de 3,0209. El valor máximo fue de 27 y el mínimo de 13.

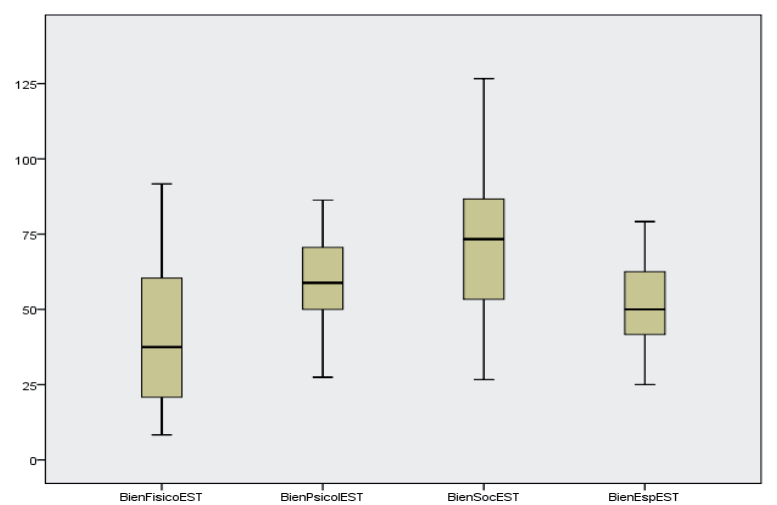

Gráfica 2. Diagrama de caja. Comportamiento estadístico básico de las dimensiones de calidad de vida Fuente: Tesis Maestría Martínez, SM. (2015)

Se identificó que las dimensiones con mayor correlación en calidad de vida son las de bienestar físico y bienestar psicológico, las cuales mostraron un coeficiente de correlación de 0,467 con una $p$ significativa de 0.01 , demostrando así que estas dos dimensiones son las que evidencian relación con calidad de vida percibida en este grupo de mujeres. Por otro lado, las dimensiones de bienestar espiritual y bienestar social mostraron una débil relación, con un valor de coeficiente de correlación de 0,010 y un valor $\mathrm{p}=0.914$, lo cual se observa en la Gráfica 2 .

La relación entre el total de calidad de vida y las dimensiones física y psicológica muestra un coeficiente de correlación de 0.715 con un valor $p$ $=0.01$ y 0.862 y un valor $p$ significativo menor de 0.01 , respectivamente, siendo significativo. Esto permite deducir que la dimensión física y la psicológica se relacionan fuertemente con el total percibido de calidad de vida.

Determinación del apoyo social en general y en sus cinco dimensiones: interacción personal, interacción social, guía, retroalimentación y ayuda tangible.

Se presentan aquí los hallazgos de apoyo social en las mujeres diagnosticadas con cáncer de mama a nivel general y con sus cinco dimensiones.

El apoyo social en mujeres con cáncer de mama, en general, no presentó simetría en sus partes. El puntaje mínimo fue de 102 puntos y el máximo fue de 234, la mediana fue de 174 puntos, la media de 168,7250, con una desviación estándar de 23,29423, tal como se observa en la Gráfica 3. 
ISSN-PRINT

1794-9831

E-ISSN 2322-7028

Vol. 15 No. 1

Ene - Jun 2018

Cúcuta, Colombia

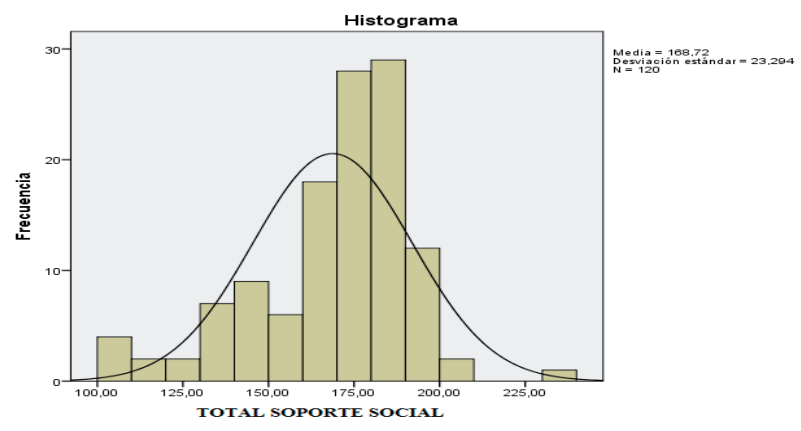

Gráfica 3. Histograma Total de Apoyo social en pacientes con cáncer de mama

Fuente: Tesis Maestría Martínez, SM. (2015)

En cuanto a la sub-escala de interacción personal, el estudio reportó una media de 48,300 con una desviación estándar de 4,2498, con un valor máximo de 60 y mínimo de 34 .

En la sub-escala guía, el rango de puntuación fue de 14 a 84 y se reportó una media de 59,73, con una desviación estándar de 10,37 y un valor máximo de 84 y mínimo de 26.

Por su parte, la sub-escala retroalimentación representó una percepción positiva de acuerdo al rango de puntuación de 6 a 36, reportando una media de 25,6 con una desviación estándar de 4,3, además de un valor máximo de 36 y un mínimo de 6.

La sub-escala de ayuda tangible evidenció una media de 13,23 con una desviación estándar de 4,3 y un valor máximo de 24 y un mínimo de 4.

La sub-escala de interacción social, con un rango de puntuación de 5 a 3 , reportó una media de 21,84 , con una desviación estándar de 5,56 y un máximo de 30 y un mínimo de 5.

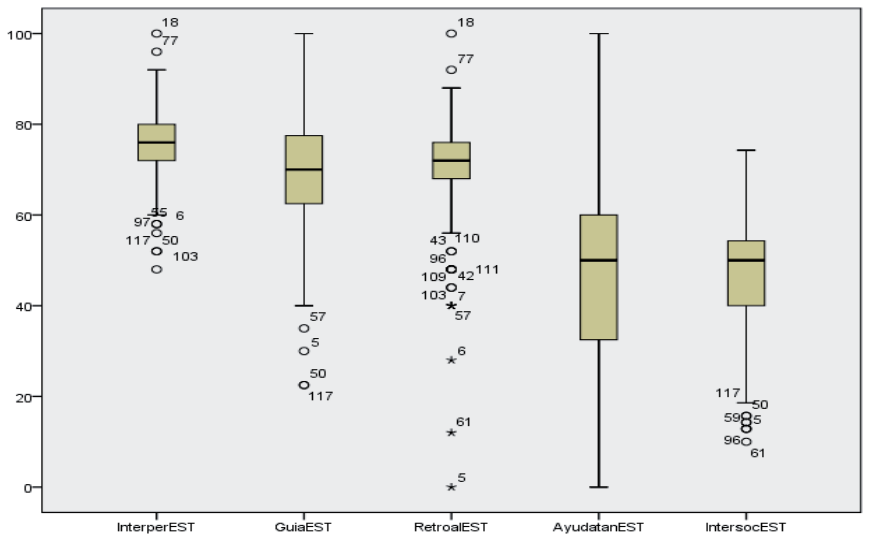

Gráfica 4. Diagrama de caja. Comportamiento estadístico básico de las dimensiones de Apoyo Social

Fuente: Tesis Maestría Martínez, SM. (2015)

La correlación rho de Spearman de las dimensiones del instrumento de apoyo social demostró una fuerte correlación entre la dimensión de retroalimentación y la de ayuda tangible, con un coeficiente de 0,674 , con la dimensión de guía, la cual mostró un coeficiente de correlación de 0,625 , y con la dimensión de interacción social, con un coeficiente de correlación de 0,553 . Además, retroalimentación y el total de apoyo social mostraron una correlación de 0,824 , permitiendo deducir que esta dimensión tuvo una fuerte relación con el apoyo social de las mujeres con cáncer de mama. Por otro lado, la dimensión de guía 
mostró una relación con la dimensión de interacción personal con un coeficiente de 0,510 y con el total de apoyo social, que mostró el mayor coeficiente de correlación $(0,840)$.

Estos datos permiten reconocer que la dimensión de retroalimentación fue la que se relacionó con dos dimensiones con un mayor coeficiente de correlación, seguida de interacción social con ayuda tangible con un coeficiente de correlación de 0,613. Es importante resaltar que todas las dimensiones mostraron una fuerte relación y un alto coeficiente de correlación, evidenciando que las mujeres que reciben diagnóstico de cáncer de mama perciben un apoyo social alto al momento de contar con una ayuda física, social, psicológica y espiritual por parte de los familiares o amigos. Esto se muestra en la Gráfica 4.

En la correlación entre la calidad de vida y el apoyo social en las mujeres diagnosticadas con cáncer de mama, mediante el coeficiente rho de Spearman se observó que aunque no fue muy alto (coeficiente de $0,284)$, llama la atención el valor p de esta correlación $(p=0,002)$, lo cual muestra significancia estadística en esta relación, tal como se observa en la Gráfica 5.

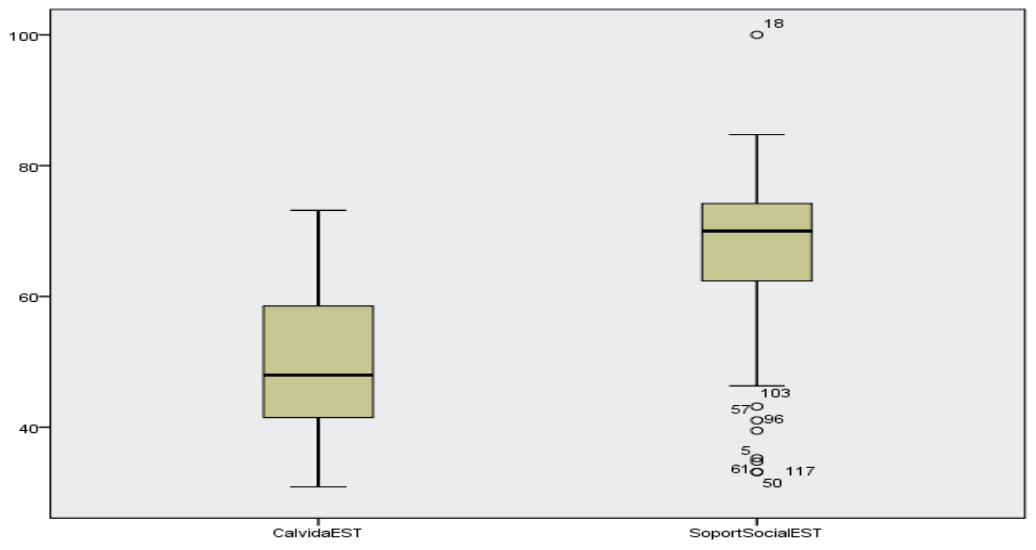

Gráfica 5. Calidad de vida y Apoyo Social

Fuente: Tesis Maestría Martínez, SM. (2015)

En cuanto a la dimensión bienestar físico de calidad de vida, el análisis mostró relación con la dimensión interacción social de apoyo social con un coeficiente de correlación de 0,299 y un valor $\mathrm{p}=0,001$, al igual que la correlación anterior; aunque el valor del coeficiente no es muy alto si es significativo el valor $P$ asociado.

Igualmente, se encontró que la correlación de la dimensión bienestar psicológico de calidad de vida mostró relación significativa con las dimensiones ayuda tangible de apoyo social -con un coeficiente de correlación de 0,224 y una p asociada de 0,014-, con la dimensión de interacción social -con un coeficiente de correlación de 0,231 y un valor $\mathrm{p}$ asociado de 0,011- y finalmente con el total de apoyo social, en donde mostró un coeficiente de correlación de 0,202 , con una significancia de 0,027 .

Por otro lado, la dimensión bienestar social de calidad de vida se correlacionó con la dimensión guía de apoyo social, con un coeficiente de 0,245 y un valor $p$ asociado de 0,007 . Así mismo, la dimensión espiritual de calidad de vida y la dimensión interacción personal tuvieron un coeficiente de correlación de 0,232 , con un valor $\mathrm{p}$ significativo de 0,011. Finalmente, la dimensión espiritual de calidad de vida y la dimensión guía de apoyo social mostró una correlación de 0,225 y un valor p asociado de 0,014 , lo cual mostró una relación significativa entre las dimensiones.

\section{Discusión}

El análisis de los datos permitió comprender que en las mujeres diagnosticadas con cáncer de mama el grupo etario con mayor porcentaje fue el de 40-59, con un $56 \%$, coincidiendo con Martins da Rosa y Vera (13), quienes dan a conocer en su estudio que el mayor número de casos nuevos, defunciones y la incidencia de la enfermedad se presentan en los grupos etarios entre 40 y 60 años, fenómeno generado por factores 
ISSN-PRINT

1794-9831

E-ISSN 2322-7028

Vol. 15 No. 1

Ene - Jun 2018

Cúcuta, Colombia reproductivos, hormonales y poca disponibilidad de servicios de detección temprana. No se puede olvidar que la edad en la que se diagnosticó a estas mujeres con cáncer de mama corresponde al periodo en el que la mayoría de ellas están activas, trabajan y son madres con hijos a su cargo.

Así mismo, los hallazgos se relacionan con lo descrito por Osma (14), en el estudio "Supervivencia a 5 años de las mujeres con cáncer de mama de Bucaramanga, donde indica que $51,3 \%$ de las edades corresponde a 54 años, lo que representa que el cáncer de mama se encuentra presente en las mujeres con edad avanzada". Aproximadamente uno de cada ocho casos de cáncer de mama se detecta en mujeres menores de 45 años de edad, mientras que dos de tres cánceres invasivos de seno se encuentran en mujeres de 55 años o más (13).

Sin embargo, es de resaltar que en este estudio el rango de edad de 24-39 años presenta un porcentaje importante de participantes (11\%), lo cual denota el hallazgo de cáncer de mama en mujeres jóvenes, situación que concuerda con la estimación hecha por Aguilar et al. (15), quienes refieren que el riesgo de cáncer de mama para el año 2020 en las mujeres de más de 25 años alcanzará a 1 de cada 3 mujeres.

En lo concerniente al estado civil de las participantes, se puede observar que la mayoría de las mujeres estaban casadas $(36,7 \%)$. El diagnóstico de cáncer, independientemente del conocimiento sobre la enfermedad, genera un impacto que no sólo afecta a la mujer, sino también a todos los miembros de la familia, haciéndose importante el apoyo familiar para enfrentar esta enfermedad. Al respecto, un estudio reciente realizado por Aguilar et al. (15) concluyó que el estado civil puede influir en el pronóstico de la enfermedad, en las mujeres casadas, porque se observa un mejor tratamiento de la enfermedad, con una supervivencia del $89 \%$ a los 5 años del inicio.

De la misma manera, el acompañamiento de una pareja (esposo) en el proceso de enfermedad contribuye al fortalecimiento de factores emocionales y sociales, lo cual ayuda a mantener un equilibrio familiar y poder, de esta manera, contribuir a una buena adaptación de la mujer durante la enfermedad y tratamiento. En similitud, Biffi y Mamede (16) dan a conocer que el apoyo familiar es fundamental para conseguir una respuesta positiva al tratamiento y para poder enfrentar la enfermedad y presentar conductas favorables que ayuden a obtener calidad de vida. Igualmente, el estudio de Marín et al. (17) sobre calidad de vida en mujeres con tratamiento de cáncer de mama en Pereira, Colombia, realizado en 2010, encontró que el $43 \%$ de las participantes eran casadas y la mayoría de ellas reportó que la calidad de vida era buena.

En cuanto a la convivencia de las mujeres, se observó que, aunque la mayoría son casadas, habitaban con otros familiares que no eran ni su esposo ni sus hijos debido a que gran parte de ellas dejó su familia principal y tuvo que convivir con otras personas para la realización del tratamiento. Se encontró que las mujeres provenían de otros municipios alrededor de la capital del departamento, siendo este el único lugar donde se cuenta con la tecnología para la realización de estos procedimientos.

El enfrentamiento de la realidad del diagnóstico, además de la vivencia de los diferentes procedimientos e intervenciones quirúrgicas estando lejos del hogar principal, reveló para ellas una serie de sentimientos de tristeza y soledad por la separación de sus seres queridos, aunque todo con el objetivo de la realización del tratamiento para "curarse" y regresar a su hogar. Calvo y Narváez (18) concluyen que "el diagnóstico y tratamiento de esta enfermedad produce en la mujer sentimientos de incertidumbre, falta de control sobre sus vidas, soledad, temor y falta de recursos para afrontar esta traumática situación y, si enfrentan estos sentimientos solas o con otra clase de familiares que no sean su familia primaria, se podrá decir que no cuentan con una red de apoyo adecuada para el acompañamiento del tratamiento". Marín et al. (17) resaltan, al respecto, que el apoyo familiar y de pareja es fundamental para sobrellevar la enfermedad. De la misma manera, la dependencia económica se vincula con otros familiares, puesto que tiene que convivir con ellos por el periodo que dura el tratamiento médico.

El $45 \%$ de las mujeres diagnosticadas con cáncer de mama no recibe ningún otro tratamiento además de la quimioterapia, denotando que este es el recurso terapéutico sistemático contra el cáncer que se viene mostrando como elección apropiada. Sin embargo, Mosher et al. (18) mencionan que el cáncer de mama y su tratamiento pueden producir una serie de problemas de calidad de vida, incluyendo la carga física, síntomas, malestar emocional, alteraciones de la imagen corporal y alteración de las actividades diarias. 
A pesar de los importantes avances en la prevención, diagnóstico y tratamiento del cáncer, así como la lucha de los entes de salud por el aumento en la cobertura de servicios y las diferentes reformas al sector salud, en el país siguen persistiendo retrasos en el acceso y atención de esta enfermedad, lo cual se ha traducido en detecciones tardías, en disminución de la sobrevida de las mujeres y, en muchos casos, en muerte. En el presente estudio se encontró que en un $36 \%$ de las mujeres con cáncer de mama, su diagnóstico se realizó en un periodo de entre 6 y 12 meses de haberse iniciado la enfermedad.

La calidad de vida en las mujeres que fueron diagnosticadas con cáncer de mama en Cúcuta presentó una estimación de buena. De la misma forma, en el estudio realizado por Marín et al. (17), la mayoría de las mujeres también reconoció la calidad de vida como buena, en controversia con Salas y Grisales (19), quienes encontraron en su estudio que la calidad de vida de estas mujeres era baja, con afectación en el bienestar social, físico y psicológico. Por consiguiente, se considera que las mujeres que padecen una enfermedad catastrófica como el cáncer pueden mantener un nivel de vida adecuado, desempeñando roles como hija, madre, esposa y profesional.

En las mujeres del estudio, el bienestar físico se encontró afectado por el sistema reproductivo y las náuseas, indicando que las afecta en algún grado tanto la enfermedad como el tratamiento, situación similar a la expresada por Ruiz y Almasa (20), para quienes la dimensión más afectada fue la física, interpretada como la de la "salud" y manifestada por la fatiga, el dolor, el insomnio, los síntomas asociados al brazo donde se encuentra el tumor y el placer sexual. De la misma manera concluye Paula (21), cuyo estudio afirma que la adaptación de las mujeres con cáncer de mama es un proceso complejo porque afecta su dimensión emocional, física, mental y social.

Así mismo, en cuanto al bienestar psicológico, como lo manifiesta Alonso y Fontanil (22) "la mayoría de las participantes manifestaron que lidiar con la enfermedad es muy difícil como resultado de la enfermedad y el tratamiento. De esta manera, el hecho de que una persona padezca cáncer modifica el sistema de relaciones sociales (familia, amigos, conocidos) establecido hasta el momento".
De acuerdo con Rincón et al. (23), "el multifactorial del bienestar psicológico, puede decirse que una enfermedad, en nuestro caso el cáncer de mama, aunque sea crónica y por tanto potencialmente limitante, no lleva implícita la condición de infelicidad en el diario vivir de la mujer. Se reconoce que la mujer que más tenga 'espíritu de lucha' es aquella que sobrelleva la situación de enfermedad, consulta más al grupo interdisciplinario y, por consiguiente, manifiesta menor deterioro psicológico y una mayor calidad de vida".

Así mismo, en lo referente al bienestar social, para este grupo de mujeres se logró establecer que recibió gran apoyo de otras personas para satisfacer las propias necesidades tanto económicas como sociales e interpersonales y, por tal motivo, no les causó aislamiento ni representó una carga económica adicional como resultado de la enfermedad. Esto demuestra que cuando se obtiene el apoyo de las personas más cercanas, se puede obtener una buena calidad de vida.

Igualmente, se logró establecer para este grupo de mujeres que las actividades religiosas proporcionaron cambios en la vida espiritual como resultado del diagnóstico, reconociendo que la paciente oncológica utiliza recursos espirituales tales como vinculación a un grupo u organización religiosa, el rezo, la lectura espiritual, la meditación o la conexión con la naturaleza. La espiritualidad es el medio que permite superar estados de la enfermedad y proporciona un sentido o propósito acerca de la vida (24).

Luxardo (25) manifiesta, en particular, que las personas ubicadas dentro de la categoría "no espirituales" tenían menores niveles de calidad de vida y de satisfacción con la vida que los grupos "espirituales".

Respecto a las correlaciones de las dimensiones de calidad de vida, se identificó que las dimensiones con mayor correlación son las dimensiones de bienestar físico con bienestar psicológico y, por tanto, se resalta que estas dimensiones están ligadas una a la otra gracias al comportamiento que existe entre cada una de ellas. Los cambios físicos que se desencadenan a consecuencia de la enfermedad y el tratamiento traen consigo sentimientos de tristeza y depresión, de acuerdo con respuestas individuales del paciente, afectando la parte relacional.
E-ISSN 2322-7028

Vol. 15 No. 1

Ene - Jun 2018

Cúcuta, Colombia 
ISSN-PRINT

1794-9831

E-ISSN 2322-7028

Vol. 15 No. 1

Ene - Jun 2018

Cúcuta, Colombia
Adicionalmente, Ferrell et al. (26) dieron a conocer que las dimensiones de bienestar físico, psicológico, social y espiritual están relacionadas con la supervivencia para mejorar la calidad de vida.

En el apoyo social, las escalas que obtuvieron un mayor puntaje fueron las de guía, retroalimentación y ayuda tangible, destacadas por el aporte de apoyo social durante la enfermedad. Por su parte, Chan (27) menciona que el "apoyo social para la población oncológica ha sido identificado como un fenómeno de tipo social por mejorar la asistencia en salud en toda la experiencia del cáncer, considerada una variable importante por contar con una fuente de apoyo emocional y tangible".

Respecto a la dimensión de interacción personal que reporta en el estudio niveles altos, se puede afirmar que las mujeres generan la aceptación de diferentes situaciones de la enfermedad, afirmando que "pueden contar con los demás si los necesitan", las personas están disponibles para escucharlas cuando quieren hablar y las consuelan cuando están molestas. Respecto a lo anterior, afirman Fachado et al. (28), el afecto y el amor son ofrecidos a los individuos por parte de su grupo más cercano.

En relación con la dimensión de Guía, las participantes manifestaron conocer cómo buscar asistencia y cómo hacer algo en una situación de dolor, presentando niveles altos, lo cual coincide con Barrera et al. (29), quienes reportaron niveles altos de guía en su estudio, afirmando que todas aquellas ayudas que percibe la persona le llegan medio de la educación y la información, siendo de gran ayuda.

Sobre la sub-escala retroalimentación, se pudo establecer que las mujeres con cáncer de mama hacen comentarios favorables cuando ejecutan recomendaciones del equipo de salud, revisan las recomendaciones que consideran importantes, revisan con el equipo de salud los consejos que se les brindan y manifiestan que la retroalimentación acerca de cómo están haciendo las cosas sin decirles si está bien o no, resulta favorable para su situación, además del apoyo y verificación de la información que les brinda el equipo de salud.

Los datos encontrados respecto a la ayuda tangible permitieron identificar que estas mujeres están parcialmente satisfechas cuando las apoyan con algunos recursos económicos, supliendo la necesidad de aportes. La situación económica de una mujer con cáncer es inestable si no cuenta con un ingreso fijo para el sustento propio y el de su familia, problema que produce desesperanza, angustia y desespero. En la mujer que genera su propio ingreso, la enfermedad y su tratamiento ocasionan ausentismo laboral continuo.

En torno a la interacción social, las mujeres de la presente investigación recibieron el apoyo de las personas más cercanas al contar con ellas para distraerse de las preocupaciones y hacer bromas o chistes para dar ánimos, fenómeno similar al expuesto por Herrera et al. (30), quienes manifiestan que las situaciones de distracción, recreación, situaciones de carácter positivo y el acompañamiento de la red de apoyo social disminuyen la soledad, el estrés y la desesperanza en estas mujeres.

En las dimensiones correspondientes al apoyo social, se encontró un alto coeficiente de correlación, demostrando que las mujeres que reciben diagnóstico de cáncer de mama perciben un apoyo social alto al momento de contar con una ayuda física, social, psicológica y espiritual por parte de parte de los familiares o amigos.

Se debe agregar que, en cuanto a la correlación de las variables calidad de vida y apoyo social se halló que no existe correlación fuerte entre ellas, lo cual se evidencia en el coeficiente de correlación Rho de Spearman de 0,284. Llama la atención el valor $\mathrm{p}=0,002$ de esta correlación, que muestra significancia estadística y es similar a lo encontrado por Medellín (31), cuyo estudio acepta la hipótesis nula de que no existe relación estadísticamente significativa entre estas dos variables. Sin embargo, este resultado entra en controversia con Vinaccia et al. (32) y Medellín y Carrillo (33), quienes reportan que la correlación en estas dos variables es positiva.

Los hallazgos encontrados en el presente estudio entre calidad de vida y apoyo social en mujeres diagnosticadas con cáncer de mama que reciben tratamiento de quimioterapia, permiten afirmar que las variables son independientes una de la otra. Sin embargo, para este grupo de mujeres tiene gran relevancia para el manejo de la salud y provoca en ellas seguridad, bienestar, tranquilidad y satisfacción, 
sentimientos que les ayudan a adaptarse a los cambios que ocurren en el proceso que están viviendo.

De acuerdo con los factores importantes que pueden explicarse desde los hallazgos de esta investigación, tales como la población estudiada, las particularidades sociodemográficas, las creencias, los factores culturales y las posibles redes de apoyo formal e informal, además de los referentes teóricos utilizados y los instrumentos, se reconoce que estos dos constructos teóricos en enfermería son multidimensionales, es decir, muchos factores intervienen en su percepción. Aunque apoyo social puede estar relacionado con esta variable, no es uno de los factores más predictivos de mayor o menor influencia en la calidad de vida.

\section{Conclusiones}

- Al establecer la relación entre calidad de vida y apoyo social en las mujeres con cáncer de mama, se acepta la hipótesis nula de que no existe relación estadística entre las dos variables. En el presente estudio, la calidad de vida en este grupo de población puede estar representada por otro tipo de variable, además del apoyo social.
- Cabe señalar que la calidad de vida de las mujeres diagnosticadas con cáncer de mama que reciben tratamiento de quimioterapia es una variable relevante para la disciplina de enfermaría, puesto que sirve para orientar las acciones hacia el cuidado de las pacientes con cáncer, teniendo en cuenta aspectos que hacen parte del manejo de la práctica en las enfermedades crónicas.

- Así mismo, el apoyo social de las mujeres del presente estudio se identificó como alto en todas sus dimensiones, demostrando con ello la adherencia de las dimensiones de ayuda física, social, psicológica y espiritual por parte de los familiares y/o amigos.

- Se recomienda, a nivel investigativo, abordar estos dos constructos -apoyo social y calidad de vida- de forma cualitativa, para poder obtener nuevos conocimientos que aporten y exploren otras formas de cuidado para esta población con cáncer y que redunden en su salud y bienestar.

\section{Conflicto de intereses}

La autora declara no tener ningún conflicto de intereses.

\section{Referencias Bibliográficas}

1. Organización Mundial de la Salud (OMS). Mundo de la Salud Cáncer. Datos y cifras, Julio 2017. [Internet]. 2017 [consultado 02 Noviembre 2017]. Disponible en: http://www.who.int/mediacentre/ factsheets/fs297/en/

2. Zaharia M. El cáncer como problema de salud pública en el Perú. Rev. Perú. med. exp. Salud pública [Internet]. 2013 [consultado 02 Noviembre de 2017]; 30(1):07-08. Disponible en: http://www.scielo. org.pe/scielo.php?script=sci_arttext\&pid=S1726-46342013000100001\&lng=es.

3. EE.UU. Breastcancer.org. Breast Cancer Statistics. [Internet]. 2013 [consultado 25 de noviembre de 2016]. Disponible en: http://www.breastcancer.org/symptoms/understand_bc/statistics

4. Clínica cancerológica de Norte de Santander. [Archivo]. Cúcuta; 2014. Estadísticas de la Ingreso egreso. Folio 23. P 26.

5. Duran De Villalobos, MM. Marco epistemológico de la enfermería. Aquichan [Internet].2002 [consultado 27 de octubre 2014]; 2(1):7-18. Disponible: http://aquichan.unisabana.edu.co/index.php/ aquichan/article/view/17/34

6. Ferrell BR, De Grant MM, Funk BM, Green SA, Garcia NJ. Quality of life in breast cancer survivors as identified by focus groups cancer. PubMed [Internet]. 1997 [consultado3 de abril 2014]; 6 (1):1323. Disponible en: http://www.ncbi.nlm.nih.gov/pubmed/9126712

7. Ferrell BR, De Grant MM, Funk BM, Green SA, Garcia NJ. Quality of life in breast cancer survivors: implications for developing support services. PubMed [Internet]. 1998 [consultado 3 abril de 2014]; 
ISSN-PRINT

$1794-9831$

E-ISSN 2322-7028

Vol. 15 No. 1

Ene - Jun 2018

Cúcuta, Colombia
25 (5): 887-95. Disponible en: http://www.ncbi.nlm.nih.gov/pubmed/9644705

8. Marín Kelso CL, Martínez Pulgarín DF, Vera Polanía F, Echeverri Rendón S, Muñoz Urbano D M. Quelal Achinchoy K, et al. Calidad de vida en pacientes en tratamiento de cáncer de mama, Pereira, Colombia 2010. Investig. andina [Internet]. 2013 [consultado mayo 21 2017]; 15(27): 811-823. Disponible en: http://www.scielo.org.co/scielo.php?script=sci_arttext\&pid=S0124-81462013000200008\&lng=en.

9. Hilbert, G. Social support in chronic illness. Cited by: STRICKLAND, Ora and WALTZ, Carolyn. Measurement of nursing outcomes: Measuring client self-care and coping skills. Volume Four. New York: Springer. 1990, p. 79-95

10. Barrera Ortiz L, Carrillo González GM, Chaparro Díaz L, Sánchez Herrera B, Vargas Rosero EP, Carreño S. Validez de constructo y confiabilidad del instrumento calidad de vida versión familiar en español. Enferm. glob. [Internet]. 2015 [citado 03 de noviembre 2017]; 14(37): 227-238. Disponible en: http://scielo.isciii.es/scielo.php?script=sci_arttext\&pid=S1695-61412015000100013\&lng=es.

11. Barrera Ortiz L, Campos deAldana MS, Carreño SP, Carrillo GM, Chaparro Díaz L, Jaimes ML, et al. Validez de constructo y confiabilidad en Colombia del instrumento soporte social en la enfermedad crónica. Repositorio. [Internet]. 2013 [consultado 20 de noviembre 2014]; 10(2):8-19. Disponible: http://repositorio.unilibrepereira.edu.co:8080/pereira/handle/123456789/146

12. Thompson J. Los principios de ética biomédica. Rev Pediatr Colombia [Internet] 2011. [Consultado 20 de noviembre de 2016]; 17(64) Disponible en: www.raco.cat/index.php/BioeticaDebat_es/article/ download $/ 257098 / 344146+\& \mathrm{~cd}=1 \& \mathrm{hl}=\mathrm{es} \& \mathrm{ct}=\mathrm{clnk}$

13. Martins da Rosa L, Vera R. Survival rates to woman with breast cancer. Review. Texto \& contexto - enferm [Internet]. 2012 [consultado 15 de noviembre 2013]; 21(4):980-989. Disponible en: http:// www.scielo.br/scielo.php?script=sci_arttext\&pid=S0104-07072012000400031\&lng=en.\%20

14. Osma, SE. Supervivencia a 5 años de las mujeres con cáncer de mama de Bucaramanga y su área metropolitana según el estadio clínico UIS, año 2013 [Tesis de maestría]. Bucaramanga: Universidad Industrial de Santander; 2013 [consultado 20 de octubre de 2015]. Disponible en: http://repositorio. uis.edu.co/jspui/bitstream/123456789/10029/2/142908.pdf

15. Aguilar Cordero MJ, Neri Sánchez M, Padilla López CA, Pimentel Ramírez ML, García Rillo A, Sánchez López AM. Factores de riesgo como pronóstico de padecer cáncer de mama en un estado de México. Nutr. Hosp. [Internet]. 2012 [consultado 04 de junio 2015]; 27(5): 1631-1636. Disponible en: http://dx.doi.org/10.3305/nh.2012.27.5.5997

16. Biffi RG, Mamede MV. Perception of Family Functioning Among Relatives of Women Who Survived Breast Cancer: Gender Differences. Rev. Latino-Am. Enfermagem [Internet]. 2010 [consultado 12 de noviembre 2013]; 18(2): 269-277. Disponible: http://www.scielo.br/scielo.php?script=sci_ arttext\&pid=S0104-11692010000200019\&lng=en.\%20

17. Marín CL, Martínez DF, Vera F, Echeverri S, Muñoz DM, Quelal AK et al. Calidad de vida en pacientes en tratamiento de cáncer de mama, Pereira, Colombia 2010. Investig andina [Internet]. 2013 [consultado 23 de noviembre 2013]; 15 (27): 811-823. Disponible en: http://www.scielo.org.co/scielo. php?script=sci_arttext\&pid=S0124-81462013000200008\&lng=en

18. Calvo Gil MJ, Narváez Pinochet PA. Experiencia de mujeres que reciben diagnóstico de Cáncer de Mamas. Index Enferm [Internet]. 2008 [consultado 19 de noviembre 2014]; 17(1): 30-33. Disponible en: http://scielo.isciii.es/scielo.php?pid=S1132-12962008000100007\&script=sci_arttext

19. Mosher CE, Johnson C, Dickler M, Norton L, Massie MJ, Du Hamel K. Living with Metastatic Breast Cancer: A Qualitative Analysis of Physical, Psychological, and Social Sequelae. The Breast Journal. [Internet]. 2013 [consultado 08 de noviembre 2013]; 19(3): 285-292. Disponible: http://onlinelibrary.wiley.com/doi/10.1111/ tbj.12107/abstract?deniedAccessCustomisedMessage=\&userIsAuthenticated=false

20. Ruiz Carreño P, Almansa Martínez MP. Calidad de vida en mujeres que conviven cuatro o más años con cáncer de mama, desde una perspectiva enfermera. Index Enferm [Internet]. 2012 [consultado 2015 de noviembre 2013]; 21(4): 190-193. Disponible en: http://scielo.isciii.es/scielo.php?script=sci arttext\&pid=S1132-12962012000300003\&lng=es.

21. Pérez P, González A, Mieles I, Uribe AF. Relación del apoyo social, las estrategias de atontamiento y los 
factores clínicos y sociodemográficos en pacientes oncológicos. Pensamiento Psicológico [Internet]. 2017 [consultado 02 de noviembre 2017]; 15(2),41-54. https://dx.doi.org/10.11144/Javerianacali. PPSI15-2.rase

22. Alonso Y, Fontanil Y, Ezama E. Apego y bienestar en mujeres en proceso de tratamiento del cáncer de mama. Anal. Psicol. [Internet]. 2016 [consultado 03 Nov 2017]; 32(1):32-38. Disponible en: http:// scielo.isciii.es/scielo.phpscript $=$ sci_arttext\&pid=S0212-97282016000100004\&lng=es

23. Rincón Fernández ME, Pérez San Gregorio MA, Borda Más M, Martín Rodríguez A. Diferencias psicológicas en pacientes con cáncer de mama según el tipo de cirugía mamaria. Cir. plást. iberolatinoam [Internet]. 2010 [consultado 20 de noviembre 2015]; 36(4): 359-368. Disponible en: http://scielo.isciii. es/scielo.php?script=sci_arttext\&pid=S0376-78922010000400009

24. Efficace F, Marrone R. Spiritual issues and quality of life assessment in cancer care Death Stud. PubMed [Internet]. 2002 [consultado 20 noviembre 2015]; 26(9):743-56 Disponible en: http://www. iss.it/binary/gend/cont/pubmed.pdf

25. Luxardo N. El cuerpo medido. Escalas y mediciones de la calidad de vida de personas con cáncer. En: IV Jornadas de jóvenes investigadores Instituto de Investigaciones Gino Germani. Buenos Aires. [Internet]. 2007 [consultado 20 noviembre 2015]; 1-16 Disponible en: http://www.aacademica. org/000-024/171.pdf

26. Ferrell BR, Grant M, Funk B, Garcia N, Otis Green S, Schaffner ML. Quality of life in breast cancer. PubMed [Internet]. 1996 [Consultado 1 marzo 2014]; 4(6):331-40. Disponible en: http://www.ncbi. nlm.nih.gov/pubmed/9128486

27. Chan CW, Hon HC, Chien WT, Lopez V. Social support and coping in Chinese patients undergoing cancer surgery. Cancer Nurs. PubMed [Internet]. 2004 [consultado1 marzo 2014]; 27(3):230-6. http:// www.ncbi.nlm.nih.gov/pubmed/15238811

28. Fachado AA, Menéndez Rodríguez M, González Castro L. Apoyo social: Mecanismos y modelos de influencia sobre la enfermedad crónica. España. [Internet]. 2013 [consultado 21 noviembre 2015]; 1(19):118-123 Disponible en: https://dialnet.unirioja.es/servlet/articulo;jsessionid=F56BE7FA8DF94 0A17C485BAB9391B052. dialnet02? codigo $=4205757$

29. Barrera Ortiz L, Campos ME, Gallardo Solarte K, Coral Ibarra R, Hernández Busto A. Soporte social percibido por las personas con enfermedad crónica y sus cuidadores familiares en cinco macro regiones geográficas de Colombia [Internet].2016 [consultado 02 de noviembre 2017]; 18(1):36-46. Disponible en: http://revistas.udenar.edu.co/index.php/usalud/article/view/2727/0

30. Herrera A, Flórez Torres I, Romero Massa E, Montalvo A. Soporte social a cuidadores familiares de personas con enfermedad crónica en Cartagena. Aquichan [Internet]. 2012 [consultado 23 Noviembre 2014]; 12(3):286-297 Disponible en: http://aquichan.unisabana.edu.co/index.php/aquichan/article/ view/2308

31. Medellín Olaya J. Soporte social percibido y calidad de vida de personas con enfermedad renal crónica sometidas a trasplante renal año 2012 [tesis de maestría] Bogotá: Universidad Nacional de Colombia 2012 [consultado 23 Noviembre 2015]; Disponible: http:/www.bdigital.unal.edu.co/8932/.

32. Puerto Pedraza HM, Carrillo González GM. Calidad de vida y soporte social en los cuidadores familiares de personas en tratamiento contra el cáncer. Revista de la Universidad Industrial de Santander. [Internet]. 2015 [consultado 24 noviembre 2015]; 47(2):125-136. Disponible en: http:// www.redalyc.org/pdf/3438/343839278004.pdf

33. Medellín Olaya J, Carrillo González GM. Soporte social percibido y calidad de vida de personas con enfermedad renal crónica sometidas a trasplante renal. av.enferm. [Internet]. 2014 Jul [citado 2017 Nov 02]; 32(2):206-216. Disponible en: http://www.scielo.org.co/scielo.php?script=sci arttext\&pid=S0121-45002014000200004\&lng=es 